\title{
Modelling Aspects of Longitudinal Control in an Integrated Driver Model:
}

\author{
Detection and Prediction of Forced Decisions and \\ Visual Attention Allocation at Varying Event \\ Frequencies
}

\author{
Bertram Wortelen", Malte Zilinski", Martin Baumann ${ }^{\dagger}$, Elke \\ Muhrer $^{\infty}$, Mark Vollrath ${ }^{\infty}$, Mark Eilers ${ }^{*}$, Andreas Lüdtke ${ }^{*}$, Claus \\ Möbus*

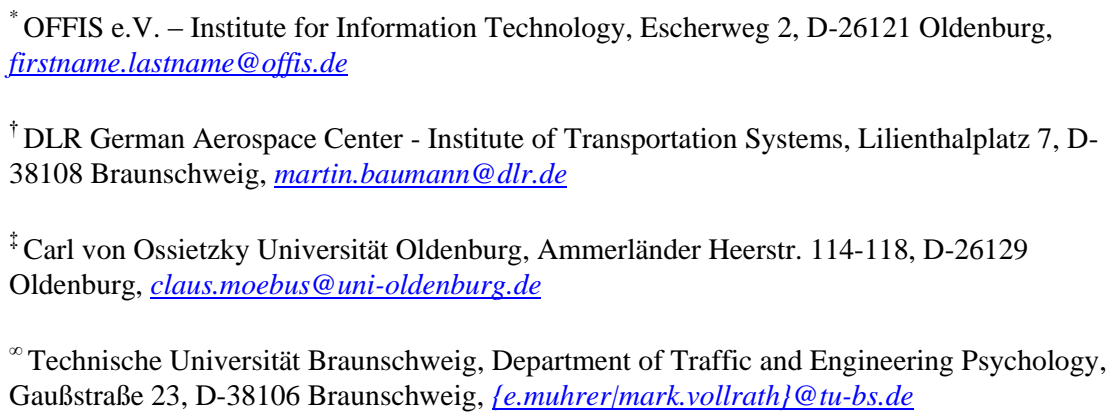

\begin{abstract}
Simulating and predicting behaviour of human drivers with Digital Human Driver Models (DHDMs) has the potential to support designers of new (partially autonomous) driver assistance systems (PADAS) in early stages with regard to understanding how assistance systems affect human driving behaviour. This paper presents the current research on an integrated driver model under development at OFFIS within the EU project ISi-PADAS ${ }^{2}$. We will briefly show how we integrate improvements into CASCaS, a cognitive architecture used as framework for the different partial models which form the integrated driver model. Current research on the driver model concentrates on two aspects of longitudinal control (behaviour a signalized intersections and allocation of visual attention during car following). Each aspect is covered by a dedicated experimental scenario. We show how experimental results guide the modelling process.
\end{abstract}

\footnotetext{
${ }^{1}$ Corresponding author

${ }^{2}$ Project Integrated Human Modelling and Simulation to support Human Error Risk Analysis of

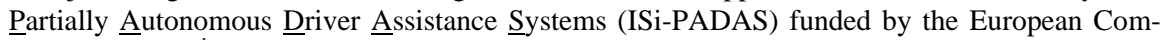
mission in the $7^{\text {th }}$ Framework Program, Theme 7 Transport FP7-218552
} 


\section{Introduction}

The number of driver support systems in cars is constantly increasing. Some of these systems influence drivers' attention and might change drivers' behaviour. Investigations on the probability of different factors to provoke inappropriate behaviour can lead to improved system designs. Driver models can help to systematically evaluate new driver assistance systems by doing closed loop simulations of drivers, systems and environments. The advantage of this simulator experiment based approach with human participants is (1) the reduction of costs for simulations and (2) to have a defined basis for comparison of design alternatives. However the main disadvantage is the incompleteness of driver models. As a matter of course they do by far not cover the great variance of human behaviour.

In a first step we create a valid DHDM without interaction to any assistance system. First results of this step will be shown in this paper. In future work the second step will be to extend the model to also handle interaction with an exemplary PADAS. Current research on our DHDM concentrates on two aspects of longitudinal control: behaviour at signalized intersections and allocation of visual attention during car following. For each aspect a dedicated experiment was conducted. We refer to the experiment for behaviour at signalized intersections with TL and for visual attention allocation with VA. Each will be described in the dedicated section. At the end we will show how the resulting models can interact by integrating them into CASCaS (Cognitive Architecture for Safety Critical Task Simulation) as executing framework, resulting in one integrated DHDM.

\section{Detection and Prediction of Forced Decision at a Traffic Light}

The TL scenario is used to investigate human driving behaviour at signalized intersection approaches. In this scenario participants approached a signalized intersection where the traffic light turned from green to yellow shortly before the driver reached the intersection. At the specific time when the yellow phase triggers drivers have finally to decide whether to stop at or to pass through the intersection. Depending on their decision different behaviour will be triggered. As shown later vehicle state and driver behaviour allow prediction of driver's decision prior to the yellow phase change.

The basic scenario was a short trial in an urban area with a single-lane priority road with low traffic density, no vehicles on the same lane, and some oncoming traffic. Pedestrians were randomly placed on the sidewalk. The whole section had a length of about $1000 \mathrm{~m}$. Behind a short curve there was a straight road of $160 \mathrm{~m}$ till the critical intersection. At this intersection was a traffic light, which switched from green to yellow in a moment, when the driver has the possibility either to pass or to stop. The traffic light was positioned $5 \mathrm{~m}$ before the intersection. The light turned from green to yellow $28 \mathrm{~m}$ before the traffic light. If the drivers 
stopped at the intersection, the traffic light switched back from red to green after 10 seconds. In the simulator, driver actions (position of gas and brake pedal) and the resulting car parameters were recorded with a frequency of $60 \mathrm{~Hz}$.

The first step for our analysis of braking decisions is to identify the relevant observable variables that allow the prediction of the future decision. Psychological motivations of drivers to find reasons why they behave like they do are not investigated.

For the prediction itself we use different classification techniques, such as Naïve Bayesian Classifier $^{3}$ (NBC), Logistic Regression, and Support Vector Machines ${ }^{4}$ (SVMs). We do not use decision trees (C4.5) since their performance in terms of AUC and accuracy are lower as stated by [2]. The selection and the size of the learning and testing data sets are systematically varied to get an overview of the prediction quality for different parameter sets. For every parameter set a crossvalidation estimates the performance of the predictive model.

The parameters recorded by the simulator were used to calculate Distance To Intersection (DTI), Time To Intersection (TTI), and a combined variable for brake/gas position. The latter variable was calculated so that it gets a positive value $[+1,0)$ when the gas pedal is pressed, a negative $(0,-1]$ for brake pedal pressed and zero when the driver did not use either pedal. The data acquired does not cover information about when and why a driver at some point in time decides to brake at the intersection.

All simulation drives have been classified in terms of approach of drivers who stop at the traffic light (brakers) and drivers who do not stop at traffic light (passers). To distinguish the dichotomic groups from each other it was sufficient to check whether the car slowed down under $5 \mathrm{~km} / \mathrm{h}$ at any time in the approach. The classification was appended to the time series and is used by the applied supervised classification algorithms for the learning and verification step.

The driver is able to see the traffic light from about $150 \mathrm{~m}$ distance. The traffic light is observable in green phase over a section of about $120 \mathrm{~m}$ while drivers approach with a mean speed of approx. $51 \mathrm{~km} / \mathrm{h}$, which equals a TTI of approximately $8-9 \mathrm{~s}$ measured $120 \mathrm{~m}$ before the intersection. After the drivers have seen the yellow traffic light and start to brake (or in the other case continue at travelling speed, in some case also accelerate), it becomes easy to differentiate between the classes braker and passer. Also before the yellow phase triggers it is possible (with a particular level of error rate) to predict the future behaviour.

A measure of the predictive quality of a model is error rate. Error rate is calculated by dividing the sum of false predictions through the sum of all cases. The prediction models applied are trained with a subset $\mathrm{A}$ of data and a different subset B to test the predictive quality with the measure of error rate. A and B are subsets of the traffic light approaches constrained by specific starting and ending points and

\footnotetext{
${ }^{3}$ See $[1]$

${ }^{4}$ See [1]
} 
$A \cap B=\varnothing$. To estimate the performance of a predictive model cross-validation technique is used.

An issue which arises in the modelling process is the selection of the data. When dealing with time series starting and ending points which represent the relevant subset have to be chosen. Grid search for different selection parameters is applied in order to find those areas with low error rates.

\section{Classification Procedure}

The following classification procedure gives an overview on how the models have been calculated and validated:

1. Discretisation of data if required by prediction model

2. Define parameters for the selection of data for grid search

a. Starting points $[-50 \mathrm{~m},-48 \mathrm{~m}, \ldots, 0 \mathrm{~m}]$ to intersection

b. Interval length $[2 \mathrm{~m}, 4 \mathrm{~m}, \ldots, 30 \mathrm{~m}]$ (defining ending points)

3. Start cross validation loop for each parameter with 10 iterations For each iteration:
a. Train model using a specific model with subset $\mathrm{A}$ of data
b. Predict class for subset B of data $(A \cap B=\varnothing)$
c. Calculate error rate

4. Calculate mean error and standard deviation of all iterations

5. Plot results

As stated by Rakha et al. [3] TTI can be used to predict the probability of braking decisions for the instant when the traffic light switches to yellow phase. For classification in the scenario at hand velocity and brake/gas pedal have been chosen for training of the prediction model. By using velocity instead of TTI no discretisation issues (discretisation is needed for some models) arise when the speed converges to zero and therefore TTI converges to infinity.

Fig. 1 shows the mean error rate of the NBC model for all subsets of the time series of the intersection approach defined by the grid search parameters. The model only uses velocity and brake/gas pedal as predictors. The $\mathrm{x}$-axis shows the starting point of the selected data $([-50 \mathrm{~m},-48 \mathrm{~m}, \ldots, 0 \mathrm{~m}])$ the $y$-axis represents the length of the interval $([2 \mathrm{~m}, 4 \mathrm{~m}, \ldots, 30 \mathrm{~m}])$. On z-axis the mean error rate for the predicted area is shown. The prediction quality improves when the distance to the traffic light reduces. But also $50 \mathrm{~m}$ in front of the traffic light the error rate is already below $25 \%$. A SVM model performs slightly better than the NBC, but the run of the curves are very similar. An advantage of the SVM in comparison to the implementation of the NBC $\left(\mathrm{e} 1071^{5}\right)$ is the fact, that no discretisation is required. Anyhow using only ten bins for each variable (equal width interval discretisation) the NBC

\footnotetext{
5 Misc Functions of the Department of Statistics (e1071), TU Wien (http://cran.rproject.org/web/packages/e1071/index.html)
} 
is a very simple model with good performance, which is capable of giving levels of certainty ${ }^{6}$ for the prediction (in contrast to $\mathrm{SVMs}^{7}$ ).

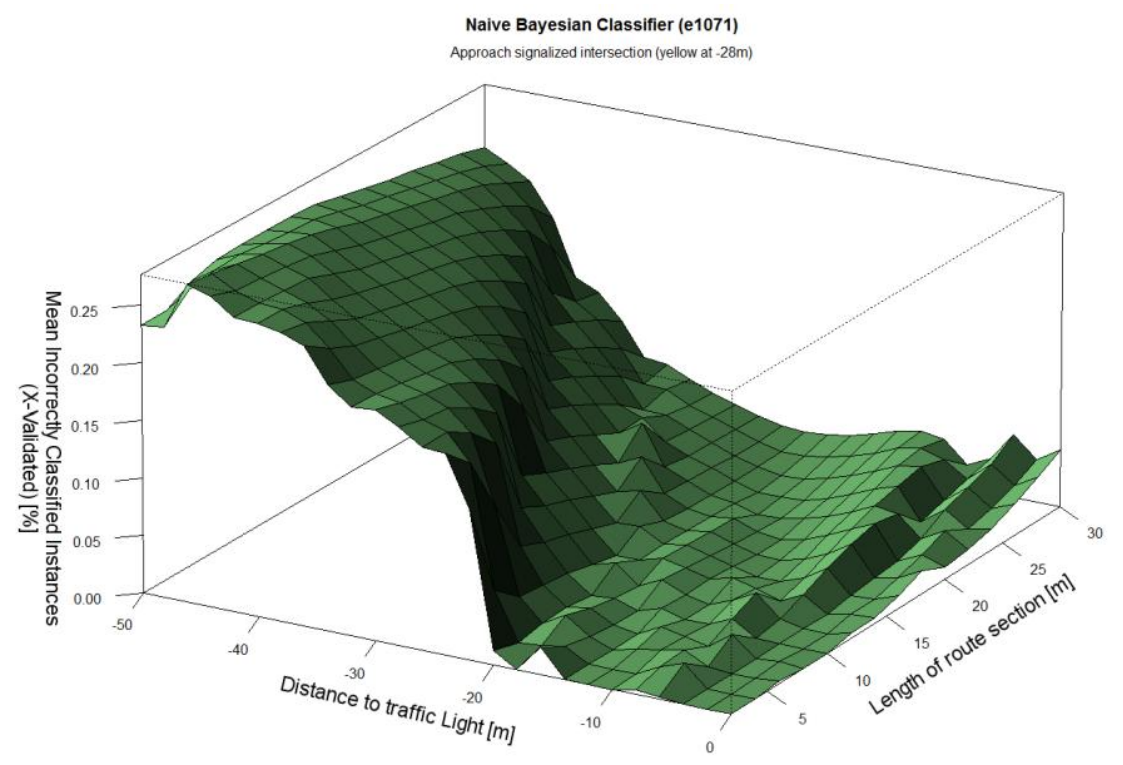

Fig. 1 Classification of braker/passer using Naïve Bayesian Classifier (Equal width interval discretisation)

\section{Visual Attention Allocation}

Visual attention allocation is a crucial part in many aspects of the driving task. In our second line of research our modelling effort concentrates on how drivers distribute visual attention among different areas of interest while executing longitudinal control.

In the following we will present layout and first results of the VA experiment that has been performed to investigate visual scanning behaviour in a car-following task. Afterwards we show how the process of visual information acquisition is modelled in CASCaS.

\section{Driving Experiments}

According to the SEEV (Salience, Effort, Expectancy, Value) model of Wickens [4], information frequency is one of the main influence factors for visual scanning

${ }^{6}$ Probability prediction is an inherent property of NBCs.

${ }^{7}$ Note: This could bypassed by using the hyperplane target function as scores. 
behaviour. To investigate the effect of different information frequencies on drivers' behaviour, driving simulator experiments in a dynamic driving simulator have been conducted at DLR [5]. For the VA experiment an urban scenario was realised in which 20 participants had to follow a leading car. The scenario consists of 24 straight road segments with a length of $600 \mathrm{~m}$, divided by intersections where the drivers had to follow the passing or turning leading vehicle. Information frequencies have been varied for two Areas of Interest (AoI): the leading vehicle and a secondary task display showing the SURT (Surrogate Reference Task) [6].

To vary the amount of information the driver perceives from the lead car, the experiment has been executed with two different speed profiles of the lead car: (1) lead car driving constantly and (2) lead car varies speed with a given pattern (see [5] for details). The amount of information for the second AoI has been varied by using different interstimulus intervals ( 3 and 6 seconds).
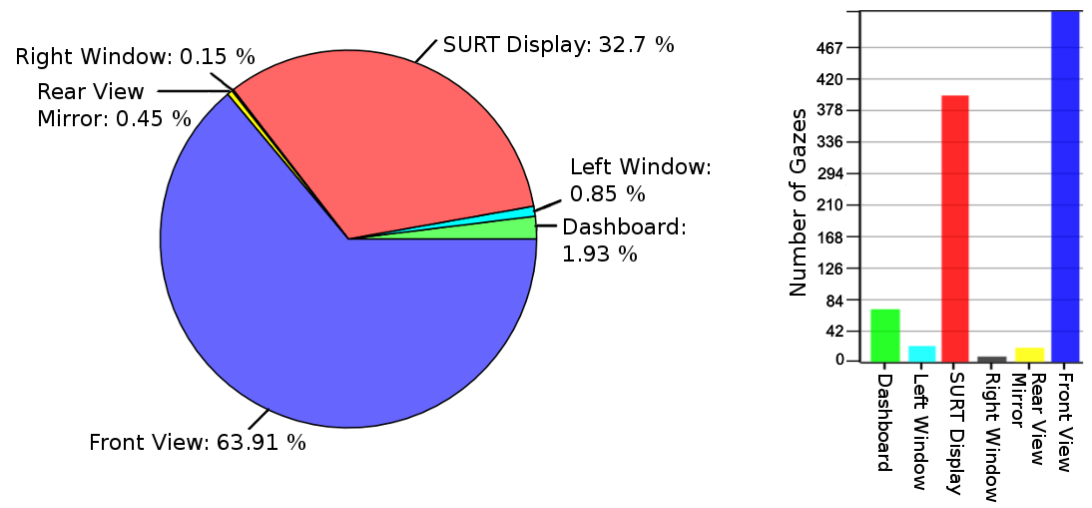

Fig. 2 Percentage Dwell Times and Gaze Frequencies for all recorded AoI's

Fig. 2 shows percentage dwell times and gaze frequencies for one participant during the complete VA experiment, independent from the experimental condition. From this it is easy to observe, that the main sources of information are the front view (for the driving task) and the secondary task display (for solving the artificial in-vehicle task).

\section{Visual Perception Process in CASCaS}

Processes involved in visual information acquisition for any model in CASCaS are very shortly described here. For a more complete description see [7].

The main influencing factor for perceiving new information for any model developed within CASCaS is the internal representation of the task to be accomplished. A task like car driving is structured for the model by a set of hierarchically organized goals. Prominent examples in the driving domain are shifting gear and keeping lane, speed or distance. For the last three of these the driver has to permanently monitor the current state of car and environment to keep the car in the lane and to keep speed and distance within an acceptable range of values. 
At any time there are several goals that the driver model has to serve simultaneously. These are referred to as active goals. The set of active goals changes over time. This is due to the fact, that new goals emerge from the driving situation or existing ones are achieved or interrupted.

The goal the model is currently working on is referred to as selected goal. As each goal normally requires different kind of information, a goal switch also means a switch of visual attention, because the model has to get relevant information for the selected goal from the environment.

CASCaS derives which information is relevant for a goal from the task description. The main formalism used for the task structure are GSM-rules (Goal, State, Mean). A GSM-rule is a 3-tuple $r=\left(g_{r}, s_{r}, A_{r}\right)$, with $g_{r}$, being the goal, which this rule shall serve, $s_{r}$ being a Boolean condition defining in which cases $r$ shall be applied, and $A_{r}$ being a set of actions to be carried out, when $r$ is applied. The condition $S_{r}$ is defined on a set of information $I_{r}$ stored in the models memory. The model sequentially selects rules, for which $\mathrm{g}$ is equal to the selected goal, and $\mathrm{s}$ holds. If no rule condition holds for the selected goal, there are two possible reasons. (1) Every information in $I_{r}$ is available for all rules associated to the selected goal, but no state description fits. In this case the model will apply no rule and just switches the selected goal. (2) The model cannot access all information elements of $\mathrm{I}_{\mathrm{r}}$. This can be, because it has not received the information yet, or it forgot the information, or due to additional timing constraints defined in $\mathrm{s}_{\mathrm{r}}$. Such constraints are used to describe the maximum allowed age for information used for the specific goal.

In the latter case when information is missing, the model will try to perceive it from the environment. Where it can find specific information is defined in a topology structure of the environment. The model questions this structure and moves its gaze to the place defined in the topology. In doing so the visual focus is driven by information demands of the selected goal (top-down-attention) and the strategy used to dynamically select goals drives the visual scanning behaviour of the model. We refer to this as the multitasking strategy. CASCaS contains also a mechanism for bottom up attention [7], which will not be further described here.

The current multitasking strategy just maintains a queue of active goals and executes them in strict sequential order. For three exemplary goals A (keeping lane), $\mathrm{B}$ (hold speed) and $\mathrm{C}$ (read navigation system) this results in static execution sequences of A-B-C-A-B-C-A-B-C-..., which is not very realistic. To overcome this we changed the multitasking strategy.

\section{SEEV Visual Attention Model}

For an initial improvement of this strategy we took implications of the SEEV model of Wickens [4], which states that there is a proportional relationship between information frequency of an AoI and probability of visual attention to it.

Besides the probability of attention the SEEV model does not state anything about fixation times and sequences on a small time scale. To account for this we changed the algorithm for goal selection. Goals are now selected on a probabilistic 
and not sequential basis. The probability of selecting a specific goal $g_{i}$ is determined by:

$$
P\left(g_{i}\right)=\frac{V\left(g_{i}\right) \cdot f\left(g_{i}\right)}{\sum_{j=1}^{n_{\text {goals }}}\left(V\left(g_{j}\right) \cdot f\left(g_{j}\right)\right)}
$$

With $\mathrm{V}(\mathrm{g})$ being the value of goal $\mathrm{g}, \mathrm{f}(\mathrm{g})$ being the event frequency of goal $\mathrm{g}$, and $\mathrm{n}_{\text {goals }}$ being the number of active goals. In this way the model accounts for both knowledge driven factors of the SEEV model. Furthermore with equal and constant $\mathrm{V}$ parameters the model is identical to the idea of the Random Constraint Sampler of Senders [8]. The event frequency can be statically assigned or can be derived from the amount of information events appearing during simulation for each goal.

Like Senders already stated the resulting model is simple and in some points unrealistic. Parameters can easily be found to fit aggregated human data like percentage dwell times on a large time scale, but it especially does not consider the effect of what Senders called rising uncertainty about the current value of the signal, which is more related to changes of attention probability on a small time scale (few seconds). At the moment we are working on a second visual attention model which also accounts for this aspect.

\section{Conceptual Integration into a Hybrid Simulation Model}

The partial driver models presented in this paper will be integrated into one hybrid driver model, using CASCaS as executing framework.

The classification model for braking prediction presented in this paper will be used in CASCaS to decide which strategy (braking / passing) the model will select. This will be done by selecting one out of a set of rules, each initiating a different strategy. The selection of a rule will be guided by the classification model.

As previously described, the visual attention model is implemented in the goal module to guide the goal selection of permanent monitoring goals.

In a subsequent step all models will be used concurrently in one driver model, to account for different aspects of the longitudinal control task of driving. 


\section{Discussion and Conclusion}

We showed how different modelling techniques can be utilised to generate partial and specialized driver models focussing on different aspects of longitudinal control behaviour. The next step will be to instantiate an integrated driver model in CASCaS as executing framework. We will investigate how such integrations can lead to a more holistic model of drivers.

Acknowledgments The research leading to these results has received funding from the European Commission Seventh Framework Program (FP7/2007-2013) under grant agreement $n^{\circ}$ 218552, Project ISi-PADAS.

\section{References}

[1] Witten, I.H., \& Frank, E. (2005). Data mining: Practical machine learning tools and techniques. San Francisco: Morgan Kaufman, vol. Second edition.

[2] Huang, J., Lu, J., \& Ling, C. X. (2003). Comparing Naive Bayes, Decision Trees, and SVM with AUC and Accuracy, Data Mining, IEEE International Conference on, p. 553.

[3] Rakha, H., El-Shawarby, I., \& Setti, J. R. (2007). Characterizing Driver Behavior on Signalized Intersection Approaches at the Onset of a Yellow-Phase Trigger, IEEE Transactions on Intelligent Transportation Systems, vol. 8, no. 3, pp. 630-640.

[4] Wickens, C.D., \& McCarley, J.S. (2008). Applied Attention Theory. Boca Raton: CRC Press.

[5] Kaul, R., Baumann, M., \& Wortelen, B. (2010). The influence of predictability and frequency of events on the gaze behaviour while driving, this issue.

[6] Mattes, S. (2003). The lane change task as a tool for driver distraction evaluation, in Quality of work and products in enterprises of the future, H. Strasser, et al., Eds. Stuttgart, Germany: Ergonomia Verlag, pp. 57-60.

[7] Osterloh, J.P., \& Lüdtke, A. (2008). Analyzing the Ergonomics of Aircraft Cockpits Using Cognitive Models, Proceedings of the 2nd International Conference on Applied Human Factors and Ergonomics.

[8] Senders, J. W. (1980). Visual Scanning Processes. 